\title{
Enhancement of Antigen-Specific Immunoglobulin G Responses by Anti-CD48
}

\author{
Dorothy Yuan Yuhong Guo Suwannee Thet \\ Department of Pathology, UT Southwestern Medical Center, Dallas, Tex., USA
}

\section{Key Words}

Cytokines $\cdot$ Immune response $\cdot$ CD48 $\cdot$ CD244 - Natural

killer cells $\cdot$ B cells $\cdot$ NP-Ficoll $\cdot$ Interferon- $\gamma \cdot$ T-independent antigen

\begin{abstract}
CD48 is a glycosylphosphatidylinositol-anchored protein expressed ubiquitously on many cell types. Despite the poor ability to signal on its own, CD48 can activate cells via interaction with its counter receptors CD2 and CD244 as well as influence the function of other cell surface molecules by costimulatory activities. We show, herein, that injection of antiCD48 antibodies into mice can augment the antibody response to a T-independent antigen, NP-Ficoll, that is representative of antigenic determinants expressed on the surface of various pathogens, such as Streptococcus pneumoniae. In C57BL/6 mice, enhancement of the response is dependent on natural killer (NK) cells as well as on the presence of CD2 and CD244, ligands for CD48, suggesting a requirement for direct interaction between NK and B cells. Interestingly, in this case, despite a similar augmentation by anti-CD48 in $\mathrm{BALB} / \mathrm{C}$ mice, the response is independent of NK or T cells, suggesting that help for this response can be derived from other innate cell types. These results provide a pathway by which CD48, when appropriately activated, can influence the course of an antigen-specific antibody response via the innate system.

Copyright $\odot 2012$ S. Karger AG, Basel
\end{abstract}

\section{Introduction}

CD48 is a glycosylphosphatidylinositol-anchored protein expressed ubiquitously on many cell types. Early experiments examining its function showed that CD48deficient mice have impaired T-cell responses upon activation [1], hypothesized to be due to alterations in costimulatory activity when cells are stimulated via the T-cell receptor [2-4]. Presumably, CD48 functions by interaction with antigen-presenting cells (APCs) via CD2, a receptor for $\mathrm{CD} 48$. However, interestingly, mice lacking CD2 do not exhibit similar deficiencies [5], possibly because CD48 can also interact with another receptor, CD244. Although CD2 is expressed on B as well as on T cells, CD244 expression is more restricted, being present on all natural killer (NK) cells but only on a subpopulation of CD8 T cells and in low density on other cell types.

CD48 itself has also been shown to have receptor activity. Crosslinking of CD48 on NK cells leads to aggregation of CD244 on the same cell, resulting in phosphorylation of tyrosine residues and subsequent activation of cytokine production [6]. In vitro experiments utilizing interleukin (IL)-2-propagated NK cells have indicated that stimulation of $\mathrm{B}$ cells by NK cells requires the presence of CD48 on B cells and leads to interferon (IFN)- $\gamma$ independent activation of downstream exons of the immunoglobulin (Ig) locus as revealed by germline transcriptions $[7,8]$. However, this activation does not result

\section{KARGER \\ Fax +4161306 1234 \\ E-Mail karger@karger.ch}

www.karger.com
(C) 2012 S. Karger AG, Basel

1662-811X/13/0052-0174\$38.00/0

Accessible online at:

www.karger.com/jin
Dr. Dorothy Yuan

Department of Pathology

UT Southwestern Medical Center

Dallas, TX 75230 (USA)

E-Mail dorothy.yuan@utsouthwestern.edu 
in productive DNA recombination, leading to the expression of functional transcripts unless the cells are also stimulated via the B-cell receptor [8]. On mast cells, CD48 itself may have receptor activity as well [9].

In order to assess the relevance of these in vitro experiments, we have examined the effect of anti-CD48 stimulation on the in vivo response to a TI-II antigen, NP-Ficoll, which can induce Ig production independent of cognate help from $\mathrm{T}$ cells but can be modulated by NK cell activity [10]. The results show that injection of antiCD48 antibodies into C57BL/6 (B6) mice prior to antigenic stimulation results in enhanced expression of IgG1 and IgG2c, responses that are otherwise relatively low. The increase is dependent on the presence of NK cells as well as on CD2 and CD244 expression. Surprisingly, despite the induction of a similar enhancement by antiCD48 in BALB/C mice, NK cells do not appear to be involved. These results show that CD48 can participate in a T-independent antigen-specific response and further implicates the non-specific help exerted by NK cells and/ or other innate cells for specific immune responses.

\section{Materials and Methods}

\section{Mice}

C57BL/6, BALB/c, BALB.NK1.1 [11], CD2 ${ }^{\text {ko }}, 2 B 4^{\text {ko }}(\mathrm{CD} 244)$ and $\mathrm{CD} 2 / 2 \mathrm{~B} 4^{\mathrm{ko}}[12]$ were bred and maintained under specific pathogen-free conditions at the UT Southwestern Animal Resources Center.

\section{In vivo Cell Type-Specific Depletions}

B6 and BALB.NK1.1 mice were depleted of NK cells by intraperitoneal injection of $75 \mu \mathrm{g}$ anti-NK1.1 antibodies on days -5 and -2 before treatment with anti-CD48. This treatment did not deplete NK T cells and kept NK cells depleted for more than 2 weeks, as determined by the retention of $\mathrm{CD}^{+} \mathrm{NK} 1.1^{\mathrm{lo}} \mathrm{NKp} 46^{-}$ cells in splenocytes. NK cells in BALB/C mice were depleted with a single injection of $20 \mu \mathrm{l}$ of anti-asialo GM1 antibodies on day -1 . T or T regulatory $\left(\mathrm{T}_{\text {reg }}\right)$ cells were depleted with anti-CD4 (125 $\mu \mathrm{g} / \mathrm{mouse}$ ), anti-CD8 (100 $\mu \mathrm{g} / \mathrm{mouse})$ or anti-CD25 (1 mg/ mouse) 2 days prior to the injection of anti-CD48. Effective depletion of each reagent was confirmed by FACS analysis of both peripheral blood lymphocytes (PBLs) and splenocytes. Anti-IFN- $\gamma$ was injected ( $75 \mu \mathrm{g} / \mathrm{mouse}) 2$ days prior to and 1 day after the injection of anti-CD48.

\section{Antibodies}

Hamster anti-CD48, control hamster Ig, rat anti-CD2 and control rat Ig were purchased from Biolegend. Anti-CD244-1 and CD244-2 were purchased from BD Pharmingen. Mouse antiNK1.1, rat anti-CD4 (GK1.5) and rat anti-CD8 (YTS) were purified from hybridoma culture supernatants using Gamma Bind (Pharmacia Fine Chemicals). Rabbit anti-IFN- $\gamma$ from serum was similarly purified. Anti-asialo GM1 was purchased from Wako
Chemicals. Anti-CD25 (PC61) was a kind gift from Dr. Bruce Blazer. All staining reagents, including anti-CD19, anti-CD4, antiCD8, anti-CD3, anti-CD69, anti-B220, anti-CD86 and SA-PCP were purchased from eBioscience or Biolegend. Anti-CD25 (7D4) was purchased from BD Biosciences. Goat anti-NKp46 and donkey anti-goat Ig was purchased from R\&D Systems. Horseradish peroxidase-conjugated antibodies for ELISA were purchased from Southern Biotechnology. All antibodies were titrated before use.

\section{FACS Analysis}

PBLs were collected on the FACScan flow cytometer and analyzed on CellQuest (BD Biosciences).

\section{Anti-CD48 Treatment and Immunization}

Two days prior to immunization, groups of 5 or 6 animals were treated with anti-CD48 dissolved in PBS. Titrations ranging from 25 to $200 \mu \mathrm{g} / \mathrm{mouse}$ yielded similar results. Therefore, most experiments utilized $50 \mu \mathrm{g} /$ mouse. $\mathrm{F}(\mathrm{ab}$ ') 2 fragments of anti-CD48 (a kind gift from Dr. Michael Bennett) also yielded similar activation patterns. Serum was collected from all animals at days 0,7 and 12 or 13 after injection with $40 \mu \mathrm{g} /$ mouse NP-Ficoll (SolidPhase Sciences) dissolved in PBS or, as indicates for one experiment, in $100 \mu$ l RIBI (Sigma).

\section{Antibody Responses}

Ig ELISA was performed as previously described [13]. The isotypes and the subclasses of bound Ig were detected by horseradish peroxidase-conjugated, isotype-specific anti-mouse Ig antibodies (Southern Biotechnology) and developed with the substrate 2,2'azino-bis(3-ethylbenzthiazoline-6-sulfonic acid) (Sigma). Results shown represent dilutions which were most sensitive to changes in antibody levels. ELISA results were evaluated by an automated ELISA reader (Molecular Diagnostics) at an optical density of $405 \mathrm{~nm}$. Statistical significance is indicated only when $p$ values were $\leq 0.05$, obtained by 1 -tailed unpaired Student's $t$ testing for the probability that the two indicated groups were similar. Similar levels of significance were obtained with analysis by the Wilcoxon 2-sample test.

\section{Results}

\section{In vivo Stimulation of NK Cells by Anti-CD48}

Our previous findings showed that ligation by antiCD48 can induce the transcription of IL-13 mRNA in IL2-propagated NK cells and that this stimulation is correlated with a relocation of one of the two ligands for CD48 and CD244, on the same cell [6]. In order to determine if anti-CD48 can stimulate NK cells in vivo, PBLs were isolated on consecutive days after injection of the antibody into B6 mice. Figure $1 \mathrm{~b}$ shows, in a representative experiment, that CD69 expression on gated NK cells (R2; fig. 1a) was significantly increased by day 2 after injection. NK cell sizes were enhanced at an even earlier time (fig. 1e). In contrast, neither peripheral blood B nor T cells exhibited detectable activation (fig. 1b). Injection of anti- 


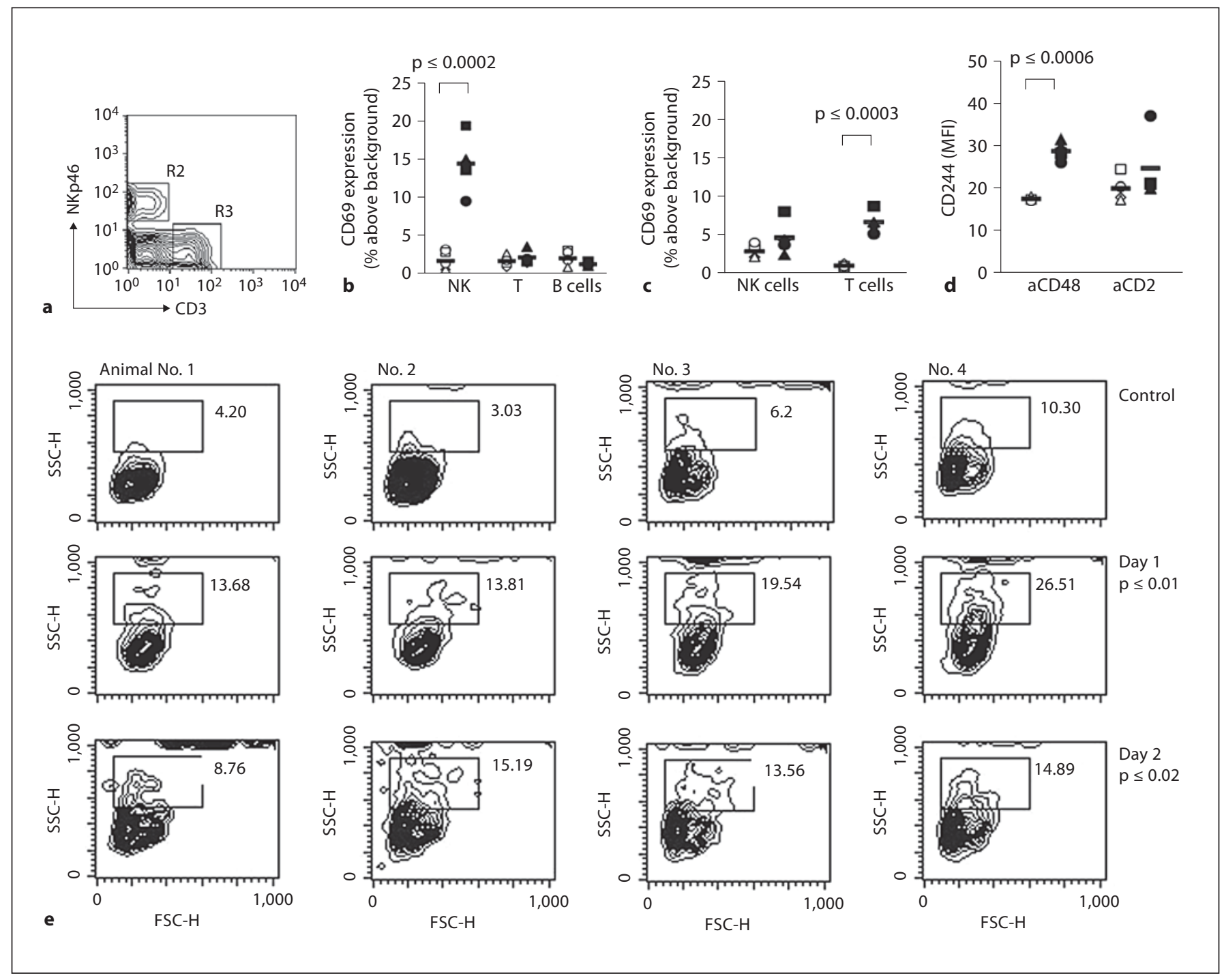

Fig. 1. a-d Activation of NK and T cells with anti-CD48. PBLs isolated from individual $\mathrm{B} 6$ mice prior to and on days 1 and 2 after the injection of anti-CD48 antibodies (50 $\mu \mathrm{g} / \mathrm{mouse})$ were stained and assessed by FACS analysis. Expression of CD69 (b) and CD244 (d) on NK (R2 in a), T (R3 in a) or B cells $\left(\mathrm{CD} 19^{+}\right)$on day 1 (open symbols) and on day 2 (closed symbols) are shown.

CD2 antibodies at the same concentration (50 $\mu \mathrm{g} /$ mouse) did not induce significant increases in CD69 expression on NK cells although T cells were activated. Interestingly, the expression of CD244 on NK cells was also significantly upregulated upon in vivo stimulation of CD48 but not by anti-CD2 (fig. 1d), whereas the expression of CD2, the other ligand for CD48, was not altered (data not shown). Therefore, anti-CD48 can activate NK cells that have not been propagated in vitro.
Another group of $\mathrm{B} 6$ mice were injected with anti-CD2 antibodies, and CD69 expression was determined in the same way (c). MFI = Mean fluorescence intensity. $\mathbf{e}$ Size profile of gated NK cells at the times indicated. Results are representative of two independent experiments.

\section{Enhancement of Isotype Switching via CD48}

To assess the functional significance of the activation of NK cells by anti-CD48, the antibodies were injected prior to antigenic challenge by the T-independent antigen NP-Ficoll. The T-independent type II response was chosen to restrict the effect to that of direct crosslinking of the B-cell antigen receptor without T-cell stimulation via major histocompatibility complex class II restricted help [for a review, see 14]. Figure $2 \mathrm{a}$ and $\mathrm{b}$ indicates in a 


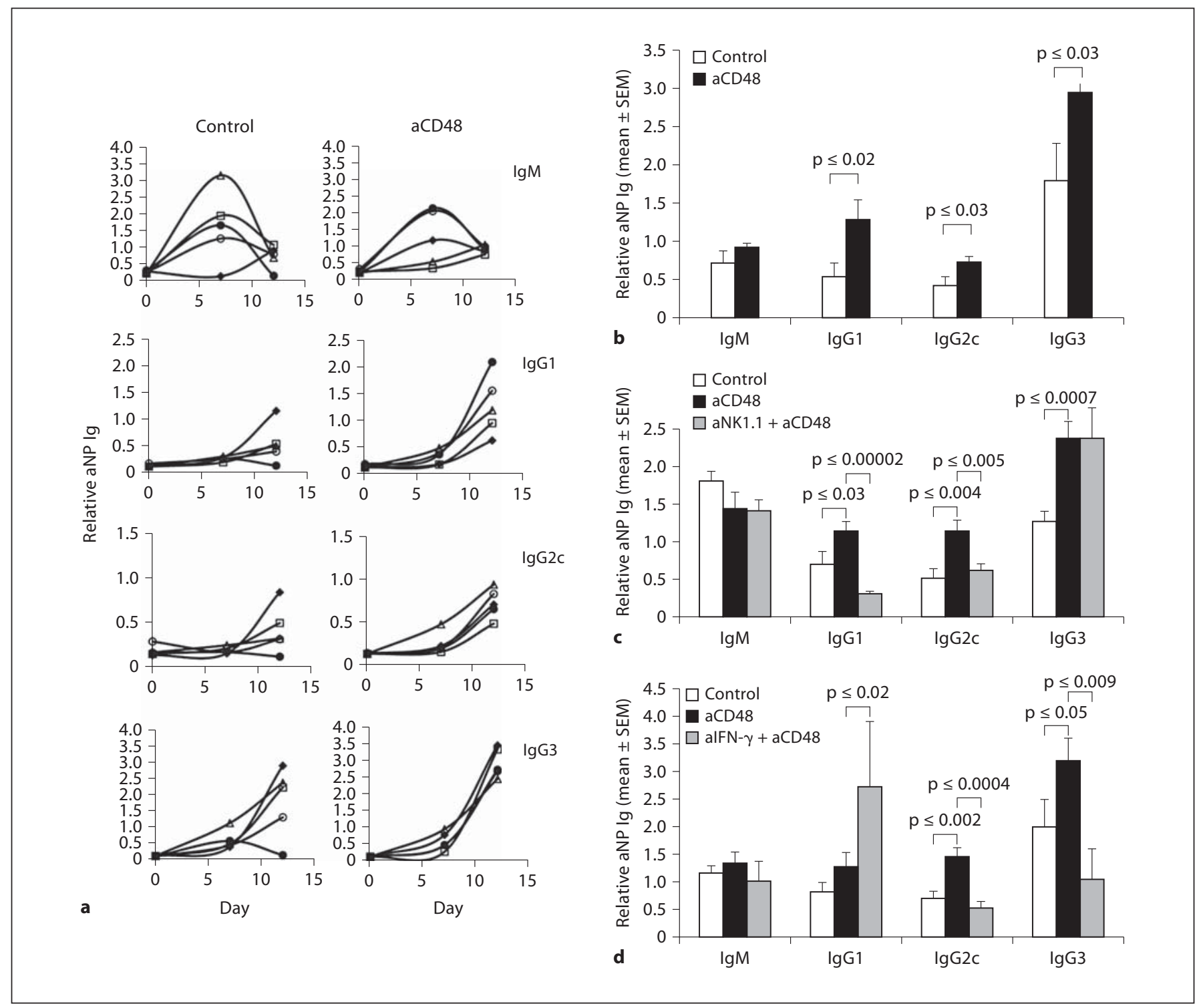

Fig. 2. NK cell-mediated enhancement of antigen-specific switch to downstream isotypes by anti-CD48 and dependence on NK cells. a B6 mice were injected with either control hamster Ig or anti-CD48 antibodies (aCD48; $50 \mu \mathrm{g} /$ mouse) 2 days prior to immunization with NP-Ficoll. Subclass-specific antigen-specific antibodies were determined on days 0,7 and 12. b Mean responses of each group are representative of two independent experiments. c B6 mice (5-6 animals per group) were injected with either control mouse Ig or anti-NK1.1 antibodies (aNK1.1) on days
-5 and -2 prior to the injection of anti-CD48 antibodies. Two days later, all animals were immunized with NP-Ficoll, and serum was collected on days 7 and 12. Mean responses on day 12 are representative of two independent experiments. d Groups of B6 mice were injected with control Ig or anti-CD48 on day -2 , or with anti-IFN- $\gamma$ (aIFN- $\gamma ; 60 \mu \mathrm{g} /$ mouse) on days -3 and -1 in addition to anti-CD48 prior to immunization with NP-Ficoll on day 0. Mean responses on day 12 are representative of two independent experiments. representative experiment that this stimulation resulted in an average more than 2 -fold enhancement in the IgG1 response and in a somewhat lower stimulation of the IgG2 $\mathrm{c}$ and IgG3 responses in B6 mice. The kinetics indicate that the IgM responses occur at earlier times, but in some animals, can diminish with time. The responses of the other classes were relatively low at day 7 but peaked at day 12 or 13 . The extent of enhancement of each isotype varied between experiments and was not dependent on the concentration of anti-CD48 injected, as similar 
results were obtained from 25 to $200 \mu$ g per mouse (data not shown).

We next investigated the role of NK cells in this enhancement since they have been shown to be responsible for the augmentation of the IgG2a/c responses to both TI-I and TI-II antigens upon stimulation by poly(I:C). NK cells were depleted prior to the injection of anti-CD48 and the subsequent challenge with NP-Ficoll. As shown in figure $2 c$, in the absence of NK cells, enhancement of both IgG1 and IgG2c, but not IgG3, responses were completely eliminated.

\section{The Effect of Anti-CD48 Is Mediated via CD2 and or} CD244

IL-2-propagated NK cells have been shown to be able to activate the germline transcription of IgG2a required for initiation of switch recombination to the downstream isotype [7]. Contact between the two cells is required for the activation that is mediated via CD2 and/or CD244 on NK cells interacting with CD48 on B cells. In order to determine if the activation by NK cells in vivo also initiates switch recombination via $\mathrm{CD} 2$, we examined the response of $\mathrm{CD} 2^{\mathrm{ko}}$ mice. Figure 3 a shows in a representative experiment that injection of the antibody failed to enhance any isotype-specific response to NP-Ficoll.

The absence of an apparent augmentation of isotype switch by anti-CD48 in CD2 ${ }^{\mathrm{ko}}$ mice could be due either to the inability of stimulated NK cells to activate B cells or to inadequate stimulation of NK cells. Since anti-CD48 induces the relocation of CD244 in vitro as well as an increase in CD244 expression, it is possible that activation via this route requires the presence of CD244. Therefore, we examined the ability of anti-CD48-stimulated $\mathrm{CD} 244^{\mathrm{ko}}$ mice to augment the NP-Ficoll response. Figure $3 \mathrm{~b}$ shows that in the absence of this ligand, the enhancement was significantly diminished. A similar lack of enhancement was found when $\mathrm{CD} 2 / \mathrm{CD} 244^{\mathrm{ko}}$ mice were stimulated with anti-CD48 (fig. 3c). In addition, figure 3d shows that in the absence of CD244, NK cells did not upregulate CD69 expression upon stimulation by antiCD48. These results correspond to our in vitro findings showing that activation of IL-2-propagated NK cells by anti-CD 48 as measured by induction of IL-13 mRNA requires the presence of CD244. Thus, the lack of an effect on $\mathrm{B}$-cell responses by anti-CD48 in CD2 ${ }^{\mathrm{ko}}$ mice is most likely due to the paucity of activated NK cells.

In view of our finding that CD244 is upregulated on NK cells upon stimulation by anti-CD48, it is possible that direct activation by anti-CD244 may increase the response to antigenic stimulation. Therefore, we injected mice with allotype-specific anti-CD244 and, as a control, also injected antibodies specific for the BALB/C allotype into B6 mice. As shown in figure 3e, neither antibodies altered the response when compared to non-stimulated animals. Therefore, stimulation via the CD244 receptor, on its own, does not impact the response to a T-independent antigen.

\section{Role of IFN- $\gamma$}

So far, our results indicate that stimulation with antiCD48 can activate NK cells and that the B-cell response to antigen can be enhanced, as revealed by increased switch recombination to downstream isotypes. Our in vitro experiments showed that activation of $\mathrm{B}$ cells requires direct interaction between NK and B cells; however, the extent of activation may be tempered by cytokines, especially IFN- $\gamma$, secreted by NK cells. To evaluate the role of IFN- $\gamma$, anti-IFN- $\gamma$ antibodies were injected prior to stimulation of the animals by anti-CD48. Figure $2 \mathrm{~d}$ shows that enhancement of IgG2c and IgG3 production was virtually eliminated in the absence of IFN- $\gamma$. However, IgG1 secretion was further enhanced. These results suggest that much of the enhancement by NK cells is mediated via a cytokine circuit which is mainly driven by IFN- $\gamma$ which can, in turn, effectively inhibit the IgG1 response. Thus, enhancement of IgG1 could have been even greater in the absence of IFN- $\gamma$-mediated inhibition.

\section{Activation by Anti-CD48 in BALB/C Mice}

The IgG1 response is generally higher in BALB/C than in $\mathrm{B} 6$ mice. Therefore, we tested the activity of anti-CD48 in $\mathrm{BALB} / \mathrm{C}$ mice to determine whether a greater enhancement can be achieved. Figure $4 \mathrm{a}$ shows that anti-CD48 can enhance both the IgG1 and IgG2a response. However, taking an average of 3 independent experiments, the extent of the enhancement was not significantly greater than the responses in B6 mice. In order to test the effect of depletion of NK cells in BALB/C mice, we utilized BALB.NK1.1 [11], in which the Nkrplb gene has been replaced by the B6 Nkrpld allele. Thus, depletion of NK cells can be readily achieved in these mice by injection of anti-NK1.1 antibodies and, as shown in figure $4 \mathrm{~b}$, NK cells remain depleted up to as long as 2 weeks. Interestingly, neither IgG1 nor the IgG2a enhancement was reduced by this treatment. Similar results were obtained when $\mathrm{BALB} / \mathrm{C}$ mice were used and NK cells were depleted by anti-asialo GM1 treatment (data not shown). Evaluation of CD69 expression also showed that NK cells were not activated by anti-CD48 stimulation, although, in contrast to the effect in B6 mice, T-cell CD69 expression was significantly increased (fig. 4c). 

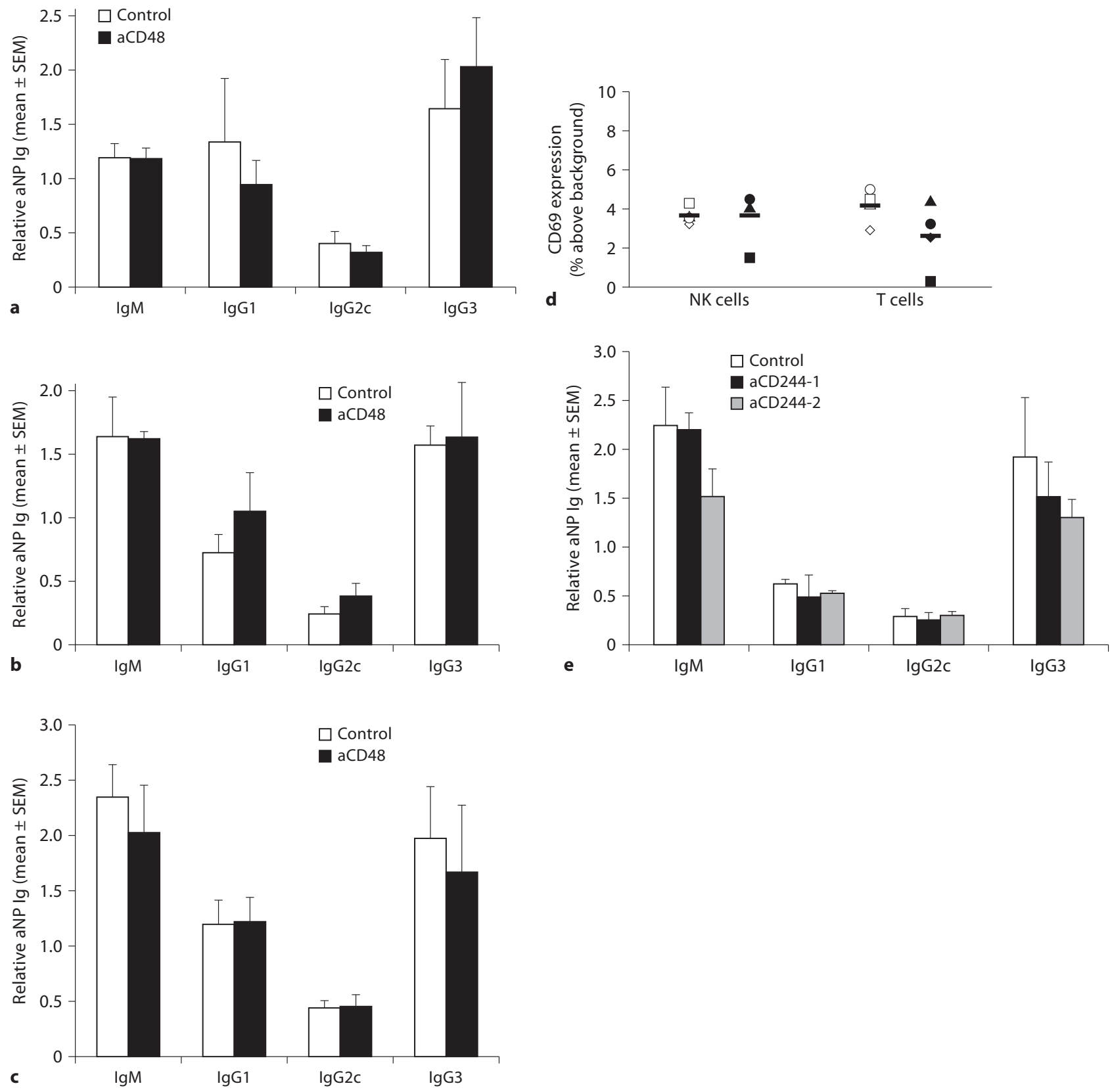

Fig. 3. Role of CD2 and CD244 in the effect of anti-CD48 (aCD48). a-c CD2 $2^{\mathrm{ko}}(\mathbf{a}), \mathrm{CD} 244^{\mathrm{ko}}(\mathbf{b})$ or CD2/CD244 $4^{\mathrm{ko}}$ (c) mice were stimulated with anti-CD48 (50 $\mathrm{gg} /$ mouse) for 2 days before stimulation with NP-Ficoll. On day 12 after antigen challenge, serum was collected and assessed for antigen-specific Ig. d Prior to (open symbols) and on day 2 (closed symbols) after anti-CD48 injection,
PBLs obtained from each of $4 \mathrm{CD} 244^{\mathrm{ko}}$ mice was assessed for CD69 expression on NK and T cells. e Groups of B6 mice were injected with allotype-specific anti-CD244-1 or CD244-2 (aCD244-1 and aCD244-2, respectively; $100 \mu \mathrm{g} / \mathrm{mouse}) 2$ days prior to immunization with NP-Ficoll. Day $12 \mathrm{Ig}$ responses were assayed together with control, non-stimulated animals. 

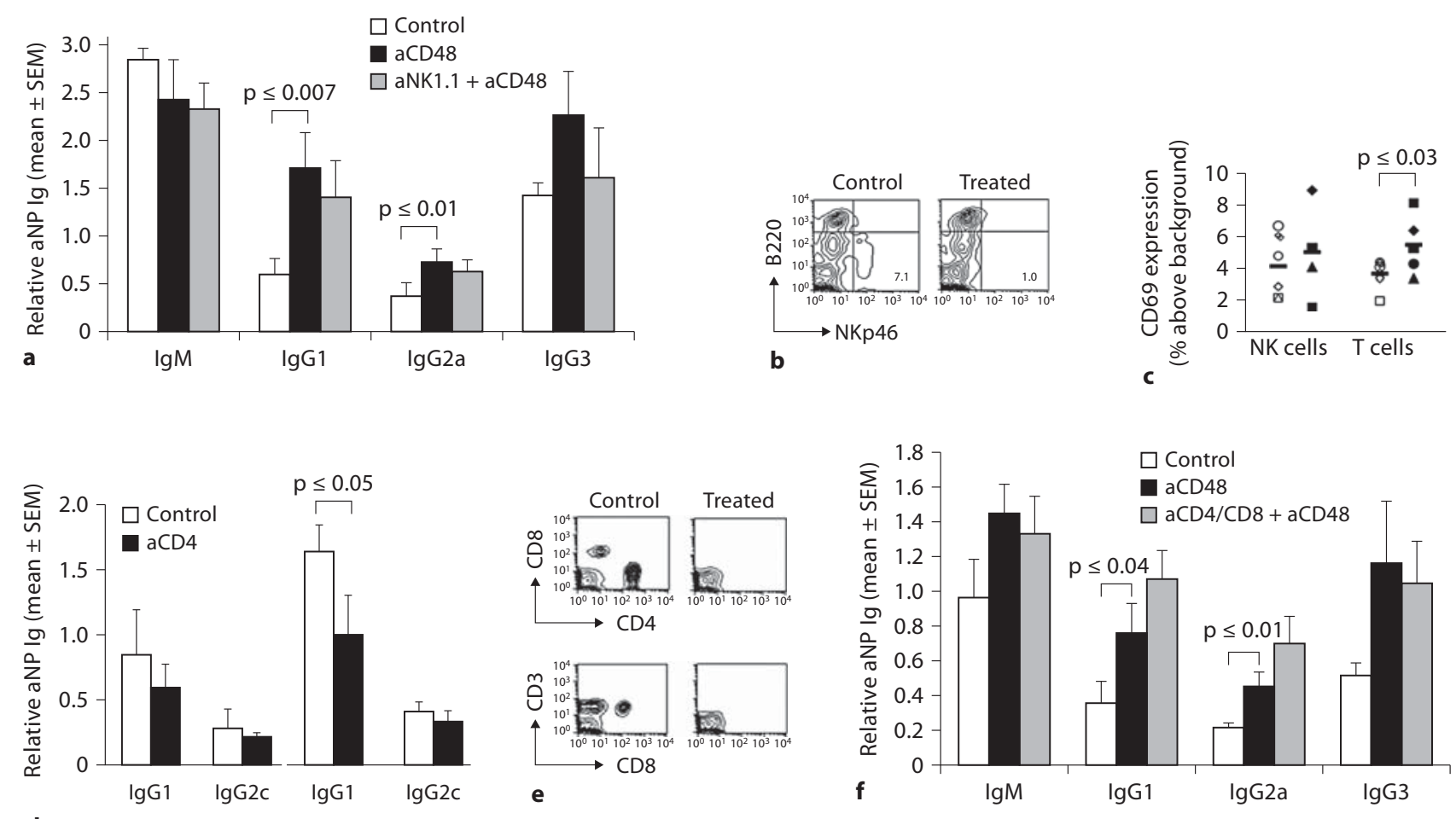

d No adjuvant Plus adjuvant
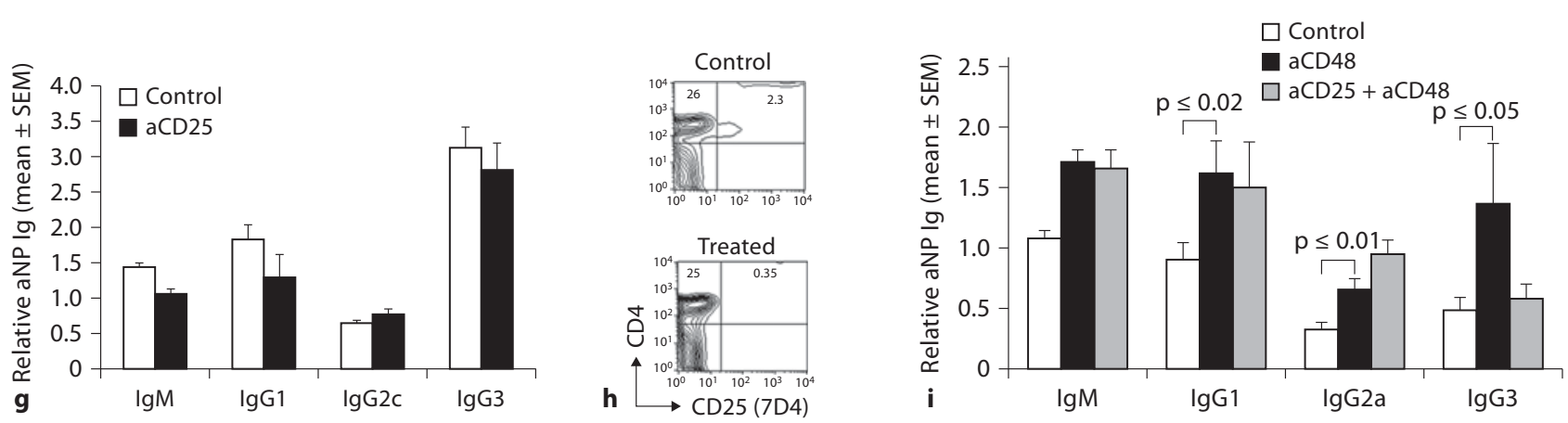

Fig. 4. Activation of $\mathrm{NK}$ and $\mathrm{T}$ cells by anti-CD48 (aCD48) in BALB/C mice. a Groups of 5-6 BALB.NK1.1 mice were injected with control antibody or with anti-CD48 2 days before immunization with NP-Ficoll. Another group was injected with antiNK1.1 (aNK1.1) on days -5 and -2 prior to the injection of antiCD48. Isotype-specific, antigen-specific Ig in serum was determined on day 7 (data not shown) and on day 13. $\mathbf{b}$ The extent of NK cell depletion was determined by FACS analysis of PBLs 14 days after injection. c CD69 levels on NK and T cells were determined on day 0 (open symbols) and day 2 (closed symbols) after anti-CD48 injection. Data presented in a and $\mathbf{c}$ are representative of two independent experiments. d BALB.NK1.1 mice were injected with anti-CD4 (aCD4; $125 \mu \mathrm{g} /$ mouse) 2 days prior to immunization with NP-Ficoll in either PBS or RIBI. Serum Ig levels were determined on day 12. e The extent of depletion by anti-CD4 and anti-CD8 (100 $\mu \mathrm{g} /$ mouse) was determined by FACS analysis of PBLs 2 days after injection. $\mathbf{f}$ Groups of BALB.NK1.1 were treated as in a, but instead of anti-NK1.1, one group was injected with a combination of anti-CD4 (aCD4) and anti-CD8 (aCD8) 2 days prior to the injection of anti-CD48 (aCD48). Serum Ig levels were determined on day 7 (data not shown) and on day 12 after antigen injection. Data are representative of two independent experiments. $\mathbf{g}$ Groups of BALB.NK1.1 mice were injected with either control antibody or anti-CD25 (aCD25; $1 \mathrm{mg} / \mathrm{mouse}$ ) 2 days before immunization with NP-Ficoll. Mean responses on day 12 are representative of two independent experiments. $\mathbf{h} \mathrm{CD} 25^{+}$cell depletion was assessed by FACS analysis of PBLs using anti-CD25 directed against an alternate determinant. i Groups of BALB/C mice were treated as in a, but instead of anti-NK1.1, one group was injected with anti-CD25 (aCD25) 2 days prior to the injection of anti-CD48 (aCD48) and 4 days prior to immunization with NPFicoll. 


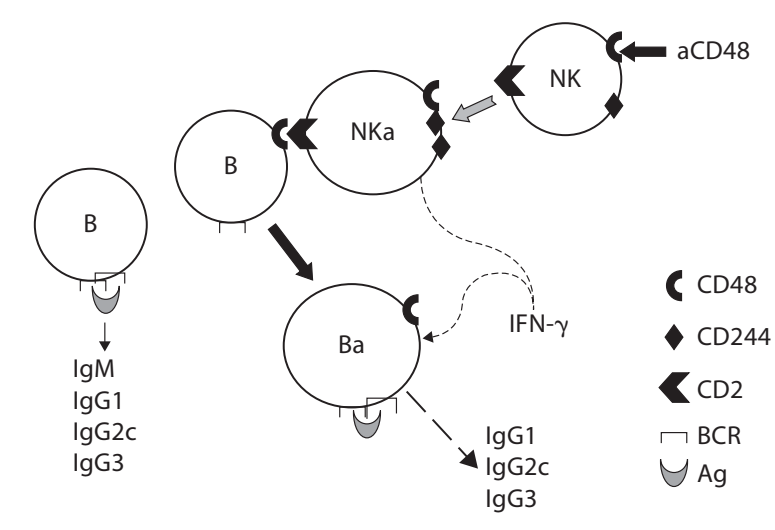

Fig. 5. Scenario for the most likely interaction between NK and $B$ cells induced by anti-CD48 (aCD48). Whereas some B cells can be stimulated by a TI-II antigen to produce a basal level of Ig, the extent of the response is low. Upon stimulation by anti-CD48, NK cells are activated, resulting in an alteration of CD244 which is required before the CD2 expressed on NK cells can become effective stimulators ( $\mathrm{NKa}$ ) of B cells via CD48. Activated NK cells can then stimulate $B$ cells that had not been activated by antigen alone. These partially activated B cells (Ba) can proceed to Ig secretion upon encounter with antigen. Whereas IFN- $\gamma$ is required for the increase in IgG2c secretion, another cytokine or, more likely, the direct activation by NK cells is sufficient for the augmentation of IgG1 secretion. This enhancement is usually somewhat inhibited in the presence of IFN- $\gamma$ production by NK cells but can be revealed by removal of IFN- $\gamma$.

Therefore, it was important to evaluate the role of $\mathrm{T}$ cells in the enhancement by anti-CD48. Since the involvement of T cells in the response to TI antigens is controversial $[15,16]$, we first confirmed the possible contribution of T cells in the response of BALB/C mice to NPFicoll. CD4 T cells were depleted prior to the injection of antigen. Figure $4 \mathrm{~d}$ shows that anti-CD4 depletion did not affect the basal response. However, when the antigen was injected in the presence of an adjuvant (RIBI), which significantly augmented the response, prior depletion of $\mathrm{CD} 4^{+}$cells can reduce the increase. This effect is likely due to $\mathrm{CD} 4^{+} \mathrm{T}$-cell-derived cytokines induced by adjuvant that can augment the response. The basal response was not affected by anti-CD8 depletion (data not shown), although effective depletion by injection of the two antibodies was confirmed (fig. 4e). To determine the role of $\mathrm{T}$ cells in the enhancement by anti-CD48, animals were injected with a combination of anti-CD4 and anti-CD8 prior to stimulation by anti-CD48. Figure $4 \mathrm{f}$ shows in a representative experiment that in contrast to the effect of
$\mathrm{CD} 4{ }^{+} \mathrm{T}$-cell depletion on the enhancement by adjuvant, enhancement by anti-CD48 was not affected.

Because the depletion of $\mathrm{T}$ cells appeared to increase, but not to a significant degree, the enhancement by antiCD48, a possible role of regulatory $\mathrm{T}$ cells is suggested. Therefore, we treated the animals with anti-CD25 antibodies (PC61) prior to immunization with NP-Ficoll. First, figure $4 \mathrm{~g}$ shows that this depletion did not affect the basal response to the antigen despite the virtually complete elimination of $\mathrm{CD} 25^{+}$cells (fig. 4f). Second, to test if $\mathrm{T}_{\text {reg }}$ cells are affected by anti-CD48, animals were depleted in a similar manner prior to stimulation by antiCD48 and antigenic challenge. The absence of $\mathrm{T}_{\text {reg }}$ cells in this case did not affect the enhancement of the response (fig. 4i). We also found that anti-CD48 did not alter the level of CD25 expression on CD4 T cells (data not shown).

\section{Discussion}

We have shown in this report that injection of antiCD48 into B6 mice can result in activation of NK cells as manifested by an increase in size scatter as well as in CD69 expression. Interestingly, CD244 expression was also enhanced upon injection of anti-CD48. The change in CD244 expression may be related to our previous finding of colocalization of CD244 upon ligation of activated NK cells. As far as can be determined, the injection did not result in alterations of activation markers in either $B$ or $\mathrm{T}$ cells, confirming previous findings that purified $\mathrm{B}$ cells were not activated by anti-CD48 in vitro [17]. Nonetheless, upon immunization with a T-independent antigen, the IgG responses were significantly enhanced. Importantly, the enhancement of both IgG1 and IgG2c can be eliminated by depletion of NK cells, and therefore, it is likely to be a functional consequence of the activation of NK cells.

The kinetics of the IgM responses (fig. 1a), although more rapid in onset, were not always maintained over time; therefore, any effect of anti-CD48 cannot be readily determined. However, it is clear that responses of all of the downstream isotypes were enhanced. Although the level of the increase was not extensive, it is in the order of that which can be achieved if mice were treated with poly(I:C) or adjuvant prior to antigenic stimulation [10, 13], effects which are also dependent on NK cells. Interestingly, the enhanced IgG3 response was not reduced upon depletion of NK cells. Notably, in a model of tumor cell-activated NK cell function which increased IgG re- 
sponses as well, depletion of NK cells did not affect the IgG3 levels [18], although the enhancement was dependent on IFN- $\gamma$. Therefore, anti-CD48 may activate other cell types that can influence the switch to IgG3. It is not surprising that the enhancement of antigen-specific IgG2c and IgG3 was dependent on IFN- $\gamma$, most likely produced by NK cells (fig. 2d). In contrast, removal of IFN- $\gamma$ prior to injection of anti-CD48 resulted in a further increase in the enhancement of the IgG1 response. We have previously shown that IL-2-propagated NK cells can induce the switch to IgG1 in vitro; therefore, NK cells activated by anti-CD48 must also be able to initiate the IgG1 response, but the level is dampened by the simultaneous production of IFN- $\gamma$ unless the cytokine is removed beforehand.

A number of reports have clearly shown that purified NK cells can directly activate isolated B cells in vitro [1921; for a review, see 22], whereas other reports have shown the involvement of NK cells in B-cell activation in vivo $[13,18,23-25]$; however, whether direct interaction between the two cell types is involved has not been established. We believe that enhancement by anti-CD48 provides the best evidence for the direct interaction between $\mathrm{NK}$ and $\mathrm{B}$ cells to be an essential component because the augmentation of Ig responses in vivo by anti-CD48 requires the presence of CD2 as well as of CD244. The requirement for $\mathrm{CD} 2$ correlates with in vitro findings that the induction of $\gamma 2 \mathrm{a} / \mathrm{c}$ germline transcription by IL-2propagated, and therefore partially activated, NK cells occurs via a direct interaction between CD2 on NK cells and CD48 serving as a receptor on B cells [9]. In this case, it was possible to show, by transwell experiments, that the interaction between the two cell types required contact. Furthermore, enhancement was also partially compromised in the absence of CD244, suggesting that colocalization with CD48 or an increased expression of CD244 is required for sufficient activation of NK cells. Interestingly, an increase has also been shown for NK cells from poly(I:C)-stimulated animals [26]. Despite the increase in expression of CD244 upon injection of anti-CD48, stimulation of mice with anti-CD244 did not result in augmentation of B-cell responses. Thus, it appears that colocalization of CD48 with CD244 required for NK cell activation does not occur with stimulation by anti-CD244 alone. This conclusion is consistent with the structural analysis of CD244 linked to CD48 on NK cells which showed distinct differences from that of the receptors on their own [27]. These results are also consistent with recent findings that the involvement of CD244 in T-cellmediated stimulation of $\mathrm{B}$-cell responses does not require
NK cells [28]. Thus, despite findings from in vitro experiments that anti-CD244 can directly activate NK cells, in vivo, the function of these cells may be dampened by the presence of CD48-expressing cells [29].

Upon consideration of these results, the best scenario for enhancement of the B-cell response to a T-independent antigen by anti-CD48 is depicted in figure 5 . Thus, it appears that upon stimulation by anti-CD 48, NK cells can be activated, resulting in the alteration of CD244 which is required before CD2 expressed on NK cells can become effective stimulators of $\mathrm{B}$ cells via interaction with CD48. This interaction results in the initiation of germline transcription of the $\gamma 1$ and $\gamma 2 \mathrm{c}$ heavy chain loci in B cells, but crosslinking of their B-cell receptor by antigen must occur before productive Ig secretion can be augmented. Whereas IFN- $\gamma$ is required for the increase in IgG2c secretion, another cytokine, or more likely the direct activation by NK cells, is sufficient for the augmentation of IgG1 secretion. This enhancement is usually somewhat inhibited in the presence of IFN- $\gamma$ production by NK cells but can be revealed by the removal of IFN- $\gamma$. It should be emphasized that the requirement for antigenic stimulation precludes the non-specific activation of B cells upon encounter with activated NK cells. An alternative explanation for the apparent activation of NK cells by anti-CD48 is that the antibody blocks inhibitory effects on NK cells by CD48 expressed on neighboring cells such that they are now more activated [30]. However, if this were the case, the basal Ig responses would have been higher in the CD $244^{\mathrm{ko}}$ mice. Similarly, because the Ig responses were not detectably lower in the knock-out mice, another possibility, i.e. that survival of activated NK cells is reduced by the absence of CD244 [31], can be discounted.

While this scenario is reasonable for the sequence of events caused by injection of anti-CD48 into B6 mice, the process by which production of downstream isotypes in $\mathrm{BALB} / \mathrm{C}$ mice is enhanced is not as easily understood. Clearly, injection of anti-CD48 can result in increases in both IgG1 and IgG2a secretion. Whereas response to TIII antigens are major histocompatibility complex class II independent, nonetheless, T-cell participation in terms of cytokine stimulation and CD40 interactions have been documented [for a review, see 14]. However, we have shown that this increase cannot be inhibited by depletion of NK, CD4 or CD8 cells. Also, enhancement cannot be attributed to changes in the status of $\mathrm{T}_{\text {reg }}$ cells since depletion of $\mathrm{T}_{\text {reg }}$ cells did not alter the production of Igs upon activation by the TI-II antigen or by anti-CD48. We have also tested for the direct activation of $\mathrm{B}$ cells or 
CD11b-positive cells in both peripheral blood and splenocytes shortly after antibody injection and have not detected increases in either CD86 or CD69 (data not shown). Nevertheless, a number of other mechanisms require further investigation. For example it is possible that antiCD48 induces the production of cytokines by a subpopulation of APCs that can affect the level of Ig secretion. Such activation has previously been shown for human cells $[32,33]$. Exogenously introduced IL-12 that induced activation of $\mathrm{B}$-cell IgG production in $\mathrm{BALB} / \mathrm{C}$ mice has been shown to be independent of both T and NK cells [34]. CD48 can also bind directly to the IL-18RA subunit which can trigger binding to IL-18RB and, in the presence of IL-18, an active signaling complex is formed [35]. Anti-CD48 has also been shown to act as an accessory molecule for the activation of human B cells via CD40mediated signals [36], possibly provided by APCs. Any of these conditions could play a more dominant role in $\mathrm{BALB} / \mathrm{C}$ than in B6 mice. Such differences in immune responses between $\mathrm{B} 6$ and $\mathrm{BALB} / \mathrm{C}$ are not unusual, es- pecially with regard to cytokine responses [37-39], and explanations have not always been straightforward [40, 41].

Regardless of the different mechanisms that may be involved in $\mathrm{B} 6$ versus BALB/C mice, the results presented herein provide evidence of a specific role for CD48 in altering the course of an immune response to a T-independent antigen, adding to the relatively limited data [42; for a review, see 43] regarding the function of this molecule.

\section{Acknowledgements}

We thank Dr. B. Blazer, University of Minnesota, for the generous gift of 7D4 antibodies. We thank Dr. M. Bennett, UT Southwestern, for providing $F\left(a b^{\prime}\right) 2$ fragments of anti-CD48. We are grateful to Dr. F. Levi-Schaffer, The Hebrew University of Jerusalem, Jerusalem, Israel, and to Dr. P.A. Mathew, University of North Texas Health Science Center, for their helpful comments regarding the manuscript. This work was supported by NIH R01 AI069253.

\section{References}

1 Gonzalez-Cabrero J, Wise CJ, Latchman Y, Freeman GJ, Sharpe AH, Reiser H: CD48deficient mice have a pronounced defect in CD4(+) T cell activation. Proc Natl Acad Sci USA 1999;96:1019-1023.

-2 Kato K, Koyanagi M, Okada H, Takanashi T, Wong YW, Williams AF, Okumura K, Yagita $\mathrm{H}$ : CD48 is a counter-receptor for mouse CD2 and is involved in T cell activation. J Exp Med 1992;176:1241-1249.

$>3$ Chavin KD, Qin L, Lin J, Woodward J, Baliga P, Kato K, Yagita H, Bromberg JS: Anti-CD48 (murine CD2 ligand) mAbs suppress cell mediated immunity in vivo. Int Immunol 1994;6:701-709.

4 Marmor MD, Bachmann MF, Ohashi PS, Malek TR, Julius M: Immobilization of glycosylphosphatidylinositol-anchored proteins inhibits T cell growth but not function. Int Immunol 1999;11:1381-1393.

$\checkmark 5$ Killeen N, Stuart SG, Littman DR: Development and function of $\mathrm{T}$ cells in mice with a disrupted CD2 gene. EMBO J 1992;11:43294336.

6 Sinha SK, Gao N, Guo Y, Yuan D: Mechanism of induction of NK activation by 2B4 (CD244) via its cognate ligand. J Immunol 2010;185:5205-5210.

7 Gao N, Dang T, Yuan D: IFN-gamma-dependent and -independent initiation of switch recombination by NK cells. J Immunol 2001; 167:2011-2018.
8 Gao N, Jennings P, Yuan D: Requirements for the natural killer cell-mediated induction of IgG1 and IgG2a expression in B lymphocytes. Int Immunol 2008;20:645-657.

$\checkmark 9$ Shin JS, Gao Z, Abraham SN: Bacteria-host cell interaction mediated by cellular cholesterol/glycolipid-enriched microdomains. Biosci Rep 1999;19:421-432.

10 Yuan D, Bibi R, Dang T: The role of adjuvant on the regulatory effects of NK cells on B cell responses as revealed by a new model of NK cell deficiency. Int Immunol 2004;16:707716.

11 von der Weid T, Beebe AM, Roopenian DC, Coffman RL: Early production of IL-4 and induction of Th2 responses in the lymph node originate from an MHC class I-independent CD4+NK1.1- T cell population. J Immunol 1996;157:4421-4427.

$\checkmark 12$ Gao N, Schwartzberg P, Wilder JA, Blazar BR, Yuan D: B cell induction of IL-13 expression in NK cells: role of CD244 and SLAMassociated protein. J Immunol 2006;176: 2758-2764.

13 Wilder JA, Koh CY, Yuan D: The role of NK cells during in vivo antigen-specific antibody responses. J Immunol 1996;156:146152.

14 Jeurissen A, Ceuppens JL, Bossuyt X: T lymphocyte dependence of the antibody response to 'T lymphocyte independent type 2' antigens. Immunology 2004;111:1-7.
15 Khan AQ, Lees A, Snapper CM: Differential regulation of IgG anti-capsular polysaccharide and antiprotein responses to intact Streptococcus pneumoniae in the presence of cognate CD4+ T cell help. J Immunol 2004; $172: 532-539$

16 Jeurissen A, Bossuyt X: T cell-dependent and -independent responses. J Immunol 2004; 172:2728, author reply 2728-2729.

17 Gao N, Dang T, Dunnick WA, Collins JT, Blazar BR, Yuan D: Receptors and counterreceptors involved in NK-B cell interactions. J Immunol 2005;174:4113-4119.

18 Koh CY, Yuan D: The effect of NK cell activation by tumor cells on antigen-specific antibody responses. J Immunol 1997;159:47454752.

-19 Snapper CM, Yamaguchi H, Moorman MA, Mond JJ: An in vitro model for T cell-independent induction of humoral immunity. A requirement for NK cells. J Immunol 1994; 152:4884-4892.

20 Snapper CM, Yamaguchi H, Moorman MA, Sneed R, Smoot D, Mond JJ: Natural killer cells induce activated murine B cells to secrete Ig. J Immunol 1993;151:5251-5260.

-21 Vos Q, Snapper CM, Mond JJ: Heterogeneity in the ability of cytotoxic murine NK cell clones to enhance Ig secretion in vitro. Int Immunol 1999;11:159-168.

22 Yuan D, Gao N, Jennings P: Interactions between B Lymphocytes and NK Cells: An Update. Berlin, Springer, 2010. 
-23 Szomolanyi-Tsuda E, Brien JD, Dorgan JE, Garcea RL, Woodland RT, Welsh RM: Antiviral T-cell-independent type 2 antibody responses induced in vivo in the absence of $\mathrm{T}$ and NK cells. Virology 2001;280:160-168.

24 De Arruda Hinds LB, Alexandre-Moreira MS, Decote-Ricardo D, Nunes MP, Pecanha LM: Increased immunoglobulin secretion by B lymphocytes from Trypanosoma cruzi infected mice after B lymphocytes-natural killer cell interaction. Parasite Immunol 2001;23:581-586.

25 Yuan D, Thet S, Zhou XJ, Wakeland EK, Dang T: The role of NK cells in the development of autoantibodies. Autoimmunity 2011;44:641-651.

-26 Assarsson E, Kambayashi T, Persson CM, Ljunggren HG, Chambers BJ: 2B4 co-stimulation: NK cells and their control of adaptive immune responses. Mol Immunol 2005;42: 419-423.

-27 Velikovsky CA, Deng L, Chlewicki LK, Fernandez MM, Kumar V, Mariuzza RA: Structure of natural killer receptor 2B4 bound to CD48 reveals basis for heterophilic recognition in signaling lymphocyte activation molecule family. Immunity 2007;27:572-584.

- 28 Brown DR, Calpe S, Keszei M, Wang N, McArdel S, Terhorst C, Sharpe AH: Cutting edge: an NK cell-independent role for Slamf4 in controlling humoral autoimmunity. J Immunol 2011;187:21-25.

-29 Kim EO, Kim N, Kim TJ, Kim K, Kim TW, Kumar V, Lee KM: Unidirectional signaling triggered through 2B4 (CD244), not CD48, in murine NK cells. J Leukoc Biol 2010;88: 707-714.
30 Lee KM, McNerney ME, Stepp SE, Mathew PA, Schatzle JD, Bennett M, Kumar V: 2B4 acts as a non-major histocompatibility complex binding inhibitory receptor on mouse natural killer cells. J Exp Med 2004;199: 1245-1254.

31 Taniguchi RT, Guzior D, Kumar V: 2B4 inhibits NK-cell fratricide. Blood 2007;110. 2020-2023.

32 Kubin MZ, Parshley DL, Din W, Waugh JY, Davis-Smith T, Smith CA, Macduff BM, Armitage RJ, Chin W, Cassiano L, Borges L, Petersen M, Trinchieri G, Goodwin RG: Molecular cloning and biological characterization of NK cell activation-inducing ligand, a counterstructure for CD48. Eur J Immunol 1999;29:3466-3477.

33 Seya T, Kasamatsu J, Azuma M, Shime H, Matsumoto M: Natural killer cell activation secondary to innate pattern sensing. J Innate Immun 2011;3:264-273.

34 Buchanan RM, Arulanandam BP, Metzger DW: IL-12 enhances antibody responses to $\mathrm{T}$-independent polysaccharide vaccines in the absence of $\mathrm{T}$ and NK cells. J Immunol 1998;161:5525-5533.

35 Fukushima K, Ikehara Y, Yamashita K: Functional role played by the glycosylphosphatidylinositol anchor glycan of CD48 in interleukin-18-induced interferon-gamma production. J Biol Chem 2005;280:1805618062.

36 Klyushnenkova EN, Li L, Armitage RJ, Choi YS: CD48 delivers an accessory signal for CD40-mediated activation of human B cells. Cell Immunol 1996;174:90-98.

37 Heinzel FP, Sadick MD, Holaday BJ, Coffman RL, Locksley RM: Reciprocal expression of interferon gamma or interleukin 4 during the resolution or progression of murine leishmaniasis. Evidence for expansion of distinct helper T cell subsets. J Exp Med 1989;169:59-72.
38 Santos JL, Andrade AA, Dias AA, Bonjardim CA, Reis LF, Teixeira SM, Horta MF: Differential sensitivity of C57BL/6 (M-1) and $B A L B / c(M-2)$ macrophages to the stimuli of IFN-gamma/LPS for the production of NO: correlation with iNOS mRNA and protein expression. J Interferon Cytokine Res 2006; 26:682-688.

39 Twine S, Shen H, Harris G, Chen W, Sjostedt A, Ryden P, Conlan W: BALB/c mice, but not C57BL/6 mice immunized with a deltaclpB mutant of Francisella tularensis subspecies tularensis are protected against respiratory challenge with wild-type bacteria: association of protection with post-vaccination and post-challenge immune responses. Vaccine 2012;30:3634-3645

40 Szliter EA, Lighvani S, Barrett RP, Hazlett LD: Vasoactive intestinal peptide balances pro- and anti-inflammatory cytokines in the Pseudomonas aeruginosa-infected cornea and protects against corneal perforation. J Immunol 2007;178:1105-1114.

-41 Ermann J, Garrett WS, Kuchroo J, Rourida K, Glickman JN, Bleich A, Glimcher LH: Severity of innate immune-mediated colitis is controlled by the cytokine deficiency-induced colitis susceptibility-1 (Cdcs1) locus. Proc Natl Acad Sci USA 2011;108:7137-7141.

42 Qin L, Chavin KD, Lin J, Yagita H, Bromberg JS: Anti-CD2 receptor and anti-CD2 ligand (CD48) antibodies synergize to prolong allograft survival. J Exp Med 1994;179:341346.

43 Elishmereni M, Levi-Schaffer F: CD48: a costimulatory receptor of immunity. Int J Biochem Cell Biol 2011;43:25-28. 